Digilec 7 (2020), pp. 85-104

Fecha de recepción: 28/02/2020

Fecha de aceptación: 13/04/2020

DOI: https://doi.org/10.17979/digilec.2020.7.0.5994

(c) (1) (2)

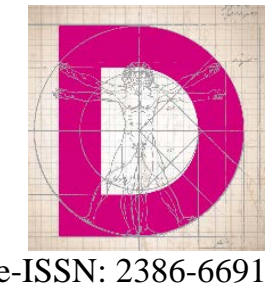

\title{
TRABAJAR LA HISTORIA LOCAL DE CÓRDOBA CON EL PROFESORADO EN FORMACIÓN DE EDUCACIÓN PRIMARIA: PERCEPCIONES SOBRE EL MUSEO ARQUEOLÓGICO
}

\author{
ADDRESSING THE LOCAL HISTORY OF CÓRDOBA WITH \\ PRIMARY EDUCATION TRAINING TEACHERS: PERCEPTIONS \\ ABOUT THE ARCHAEOLOGICAL MUSEUM
}

\author{
Rafael GUERRERO ELECALDE* \\ Universidad de Córdoba. \\ Orcid: orcid.org/0000-0003-4271-897X \\ Miguel Jesús LÓPEZ SERRANO* \\ Universidad de Córdoba. \\ Orcid: orcid.org/0000-0002-3953-1998
}

\section{Resumen}

En estas páginas se encuentran algunos de los resultados de una investigación llevaba a cabo con el alumnado de tercero del Grado de Educación Primaria de la asignatura de Didáctica de las Ciencias Sociales, de la Universidad de Córdoba, durante el curso académico 2019/2020. Se pretende conocer la percepción del futuro profesorado sobre el valor del Museo Arqueológico como recurso didáctico. A nuestro parecer, es una herramienta útil para el conocimiento del entorno más cercano y para el aprendizaje de los diversos procesos históricos, con el establecimiento de un diálogo entre lo particular y lo general, lo concreto y lo abstracto y fenómenos locales con fenómenos globales. Asimismo, se prestó atención a la didáctica del patrimonio, elemento que interrelaciona directamente el medio y la vida de nuestro alumnado. Todo ello con la intención de optimizar la calidad de los procesos de enseñanza-aprendizaje de las Ciencias Sociales. Para ello, antes y después de su realización, se preguntó a los futuros docentes sus impresiones y perspectivas sobre estas cuestiones con el objetivo de recoger respuestas y también interrogantes que hagan reflexionar sobre los contenidos y el diseño de la docencia.

Palabras clave: Museo Arqueológico; historia local; patrimonio; Educación Primaria; Ciencias Sociales.

\footnotetext{
Abstract

In these pages there are some of the results of a research carried out with the third-year students of the Primary Education Bachelor Degree of the subject of Didactics of Social

* Depto. Didácticas de las Ciencias Sociales y Experimentales. Facultad de Ciencias de la Educación. Avd. San Alberto Magno, s/n. 14071 Córdoba. Email: rgelecalde@uco.es; mjlopez@uco.es
} 
Sciences, of the University of Córdoba, during the academic year 2019/2020. The aim is to know the perception of future teachers about the value of the Archaeological Museum as a didactic resource. In our opinion, it is a useful tool for understanding the closest environment and for learning the historical processes, establishing a dialogue between the particular and the general, the specific and the abstract, and local with global phenomena. Likewise, attention was paid to the teaching of heritage, an element that directly interrelates the environment and the life of our students. All this with the intention of optimizing the quality of the teaching-learning processes of the Social Sciences. To do this, before and after it was carried out, future teachers were asked about their impressions and perspectives on these questions in order to collect answers and also to make them reflect on the content and design of teaching.

Key Words: Archaeological Museum; local history; heritage; Primary Education; Social Sciences. 


\section{INTRODUCCIÓN}

Como formadores de los futuros docentes de Primaria en la ciudad de Córdoba, hemos creído necesario intentar buscar respuestas que nos ayuden a mejorar la calidad de los procesos de enseñanza-aprendizaje de las Ciencias Sociales a través de una investigación que nos permita optimizar las tareas de enseñanza-aprendizaje a través de la percepción que tienen los futuros docentes sobre la actividad docente planteada. Por este motivo, nos ha parecido relevante presentar al alumnado de la asignatura de Didáctica de las Ciencias Sociales, de tercer curso de Educación Primaria de la Universidad de Córdoba (UCO), la historia local, tan relacionada con el medio donde desarrolla su vida el alumnado, como recurso para el aprendizaje de la Historia, Geografía y Patrimonio. A nuestro entender, puede ser un vehículo para el conocimiento, la comprensión, y el rendimiento escolar y ayudar a potenciar las habilidades de lectura, así como de la investigación por parte del alumnado (Clío 92, 1995: 22-27; Pedreño Ros, 2015, p. 53).

Los trabajos de historia local permiten interesantes posibilidades para la enseñanza de las Ciencias Sociales. Por una parte, posibilita al alumnado entender que los vestigios del pasado que se conservan a nuestro alrededor son fuentes de primer valor la comprensión y aprendizaje de la Historia.

Igualmente, favorece conocer que el trabajo, las habilidades y las técnicas del conocimiento de estos restos históricos son debidos a la aplicación de un método de investigación de carácter científico, que permite colocar los resultados del aprendizaje en un contexto general. Por otra parte, puede iniciar al alumnado en la interpretación histórica, con la reconstrucción de los acontecimientos y con la incorporación de los conceptos teóricos adecuados. Por último, la didáctica de la historia local animará la curiosidad por la exploración histórica del entorno, activando el interés por las ciencias sociales y su construcción (Pineda-Alfonso, 2018).

En definitiva, entendemos que la historia local es una herramienta muy útil para establecer un diálogo entre mundos diferentes que se encuentran interconectados: lo particular y lo general, lo concreto y lo abstracto y fenómenos locales con fenómenos comunes (García de Miguel, 2012; Clío 92, 1995: 22-27; Pedreño Ros, 2015, p. 53).

En este contexto, y entre todos los recursos para la enseñanza de la historia local, hemos elaborado una investigación con el antedicho alumnado del Grado de Educación Primaria relacionada con el Museo Arqueológico de Córdoba, teniendo el convencimiento de que los museos locales y provinciales son lugares especialmente interesantes para este fin, ya que también son conservadores del legado histórico y patrimonial de la localidad (Serrano, 2018; Estepa, Jiménez, Martín, Wamba \& Cuenca, 2012; Fernández, 2003; Pol, 2001; López Arroyo, 2014).

El Museo Arqueológico de Córdoba se encuentra situado en pleno casco histórico de la ciudad, por lo que es de fácil acceso para nuestro alumnado. Además, guarda de gran tradición en esta capital andaluza, ya que desde 1960 su sede principal está ubicado en el palacio de la familia Páez de Castillejo. En los últimos años, el museo fue ampliado 
con la incorporación de otro edificio, donde actualmente se hallan los mayores espacios expositivos.

Durante su visita, se puede realizar un recorrido por la historia de Córdoba y su provincia desde dos puntos de vista. A través de un itinerario cronológico (que transcurre desde la Prehistoria hasta la Edad Media) y por otro de carácter temático (dirigido a mostrar el mestizaje cultural propio de la construcción de la ciudad), donde las exposiciones están organizadas en estos apartados: "Córdoba y su territorio”, "Córdoba, poder y centralidad” y "Vivir en Córdoba”.

Asimismo, y como elemento de gran reconocimiento pedagógico para la enseñanza de las Ciencias Sociales, el Museo de Córdoba también es un yacimiento arqueológico: en su solar fue encontrado el teatro romano de la ciudad que, tras haber sido preparado adecuadamente, está abierto al público en su sótano, por lo que se puede visitar restos de su amplio graderío.

En los últimos tiempos, los museos se están renovando de forma importante con el deseo de ganar visitantes y de implementar sus competencias educativas, estableciendo nuevos métodos expositivos y variados recursos didácticos, con el objetivo de que sean utilizados por el profesorado tanto en el interior de sus instalaciones como en la propia escuela (Gesché-Koning, 1998; Belarte, 2004; Fernández, 2003; García Díaz, \& Martín Marcos, 2009, Bonilla Martos, 2017). En la actualidad, uno de los objetivos de los museos es la didáctica y así lo confirma el Consejo Internacional de Museos (ICOM), que entiende el museo como una institución permanente "que adquiere, conserva, investiga, comunica y exhibe para fines de estudio, de educación y de deleite, testimonios materiales del hombre y su entorno". Como vemos, ahora se comprenden los museos como un espacio comprometido hacia el servicio y desarrollo de la sociedad, por lo que su dimensión educativa está aquí perfectamente insertada y es el propio ICOM quien trabaja para que los gobiernos tengan como prioridad principal fomentar una mayor relación entre los programas escolares y las actividades museísticas (Serrano Moral, 2018, 112118).

A pesar de estas tendencias, y en general, los planes de estudio universitarios no guardan un apartado específico para la formación en educación patrimonial y, cuando lo hacen, es de un modo anecdótico, sin un interés concreto por su desarrollo. De este modo, se puede contemplar el poco nexo existente, dentro de las concepciones del futuro profesorado, entre patrimonio y ciudadanía (Cuenca, 2004). Respecto a nuestro caso, en la Universidad de Córdoba, a lo largo de los cuatro años del grado de Primaria, no existe una asignatura dedicada al patrimonio, aunque sí existe una dedicada en el grado de Educación Infantil.

En definitiva, y para trabajar sobre estas cuestiones, intentamos ofrecer una reflexión sobre las posibles aportaciones de la investigación en esta práctica docente, entendiendo que la principal valía que caracteriza la investigación es la mejora de la realidad en la que cada uno ejerce la docencia. Así pues, desarrollamos una investigación para conocer las percepciones del alumnado del Grado de Primaria que cursaba la asignatura de Didáctica de las Ciencias Sociales en relación a la enseñanza de la historia de local a través de una visita didáctica en el Museo Arqueológico de Córdoba (López Serrano \& Guerrero Elecalde, 2019). Con ello, se decidió plantear que el patrimonio 
histórico de la ciudad y de la región se convirtiera en un vehículo principal para la formación de los futuros docentes. Asimismo, también se estableció como objetivo la sensibilización del alumnado sobre la riqueza de los museos como recurso didáctico y hacerles partícipes de las posibilidades de su uso y aplicación como futuros docentes.

\section{ANTECEDENTES Y FUNDAMENTACIÓN TEÓRICA}

Hace ya unas décadas que el profesor Joaquim Prats publicó un valioso trabajo donde reflexionaba sobre el debate generado con respecto a la historia local como opción didáctica (Prats, 1996), que nos ha servido de inspiración para desarrollar esta investigación con el alumnado en formación. Además, en este estudio, realiza una excelente revisión de los diferentes momentos que ha vivido la historia local como fundamento y recurso para la enseñanza de las Ciencias Sociales. Reflexiones que ayudan a comprender mejor las bondades y la problemática que presenta la enseñanza de la historia local.

A finales del siglo XIX, ya se planteó la necesidad de introducir la historia local como herramienta de aprendizaje en la Educación Primaria, ya que se entendía que el alumnado sólo tenía la capacidad de comprender los acontecimientos cercanos. Esta percepción, contrarrestaba profundamente con las políticas establecidas en aquella época por los estados nacionales, que estaban utilizando la enseñanza de la Historia como una herramienta ideológica de construcción nacional y de determinados ciudadanos patrióticos.

Desde los años 1950, en los países anglosajones se fue introduciendo la historia local en los programas de estudio, aunque no fue así en el resto de los países europeos (como Francia o Alemania), quedando al margen de los procesos de enseñanza. En aquellos territorios prevaleció la idea de que su aprendizaje no favorecía ni el arraigo ni la motivación entre el alumnado. En España, salvo algunas experiencias en la Segunda República, continuaron con esta tendencia europea.

En cambio, a partir de los años 1970, se generalizó la pedagogía del medio y, desde entonces, los estudios del entorno, en los que estaban incluidos la historia local, fueron la piedra angular de los contenidos que se incluyeron en el currículum. En España, fueron los grupos de renovación pedagógica de profesores de EGB los que apoyaron la integración del estudio del medio en Primaria. Para este colectivo, los conocimientos históricos formaban parte de los contenidos sociales porque, siguiendo los presupuestos de Piaget, a estas edades únicamente posible trabajar contenidos concretos. De este modo, entendían que los estudios de historia local establecen puentes entre lo concreto, lo conocido y lo cercano y el aprendizaje de conceptos más complejos. Asimismo, se presentaba como el procedimiento ideal para trabajar aspectos relacionados con la investigación, como la formulación de hipótesis, la verificación y síntesis del conocimiento, por ejemplo. Con la LOGSE, los estudios de historia local y del medio fueron principales en Educación Primaria, si nos detenemos en el área de las Ciencias Sociales. 
Con el paso del tiempo, y de las nuevas políticas, esta tendencia ha quedado algo olvidada, como también la enseñanza de la historia local, que ha quedado relegada en la Didáctica de las Ciencias Sociales tras otros contenidos disciplinares, que cada vez son más parecidos a los modelos tradicionales (Prats, 1996).

En este punto, parece necesario volverse a plantear los valores de la enseñanza de la historia local, su implantación en las aulas, así como probar nuevos recursos que ayuden a su desarrollo. Y es que una buena observación desde perspectiva local favorece un acercamiento a la materialidad espacial de las grandes construcciones sociales, así como al conocimiento de su dinámica interna. Igualmente, ayuda a un escudriñamiento en los orígenes de las estructuras, junto con su devenir diario, además de otorgar una información concreta sobre los modelos generales (Pedreño Ros, 2015: 50). Por otra parte, no debemos olvidar que comprometer al alumnado en la construcción y apropiación de su historia local les posibilitará construir identidades y una mayor comprensión de la Historia, como una de las bases principales para el aprendizaje de las Ciencias Sociales.

A la vez deben existir recursos accesibles e idóneos para que el alumnado los pueda entender en toda su extensión. En este punto, los museos parecen mostrarse como un recurso valioso para la enseñanza de la historia local. Como se ha planteado anteriormente, el concepto de museo está cambiando profundamente. Ya no se trata de un espacio dedicado exclusivamente a la conservación, protección, restauración y exposición de los elementos patrimoniales, sino que su concepción es más amplia, donde el visitante se convierte en un elemento principal para establecer una relación bidireccional entre los elementos patrimoniales y la comunidad (Fontal, 2003).

En esta línea, cabe perfectamente una estrecha relación entre los museos y la escuela $\mathrm{y}$, como plantean diversos autores, estos espacios son contextos no formales ideales para el desarrollo del aprendizaje (Cole, 1999; Rinaudo, 2007). Respecto a la educación patrimonial, la escuela no puede ser el único ámbito de enseñanza a pesar de su papel primordial debiéndose establecer nuevos caminos para su mejora. Por este motivo, los museos se convierten en ámbitos educativos especiales para el aprendizaje, así como recursos muy útiles para el desarrollo del trabajo procedimental (Asensio \& Pol, 2002; Santacana, 1998; Lucas \& de Alba, 2017).

Creemos que es muy necesario implicar a los docentes (y a los profesores en formación) con los espacios museográficos (Krechevsky et al., 2000), para que los visiten y los observen como recursos de aprendizaje. Asimismo, habría que mejorar su formación en el ámbito de la educación patrimonial (Fontal, 2003). Por ello, se debería desarrollar, a lo largo en la formación del profesorado, competencias relacionadas con el conocimiento e interpretación de la realidad como un sistema complejo, donde el patrimonio puede servir como fuente de información del pasado y referente identitario cultural del presente y del futuro: desarrollo del pensamiento crítico y reflexivo, y las fuentes patrimoniales ayudan al análisis y la interpretación de los fenómenos sociohistóricos; implicación a la comunidad, y el patrimonio permite comprometer a la sociedad; comprensión del futuro a través del análisis del pasado, como bien lo muestra los elementos patrimoniales; y desarrollo el proceso de enseñanza-aprendizaje empleando el patrimonio como objetivo, contenido y recurso en la didáctica de las Ciencias Sociales (Estepa, Cuenca y Martín, 2011; Valbuena, 1994). 
Igualmente, la apropiación del patrimonio histórico de la localidad por parte de las personas ayuda a la creación y la consolidación de una identidad ciudadana responsable. Eso es posible gracias al fomento del respeto y de conservación del entorno, el interés por acrecentar la capacidad de comprender y resolver las problemáticas existentes, y la disposición por responsabilizarse del mismo, como verdaderos protagonistas de su conservación y divulgación (Hernández Cardona, 2005; Muñoz Martínez, 2006; Iglesias Curto, 2017; Aguado, 2018; Gil Carmona, 2018).

El museo es recurso muy válido para la enseñanza. La realización de visitas didácticas, a semejanza de lo que ocurre con los itinerarios, se presenta como una herramienta muy valiosa para el aprendizaje del patrimonio y, como no, de la historia local. En su desarrollo, y entre otras materias, impulsa la comprensión, incentiva la curiosidad, la investigación y el descubrimiento y favorece escapar de la rutina, educar en valores, así como el trabajo colaborativo (Liceras, 2018).

También puede ser una técnica motivadora y atractiva para trabajar contenidos, entender e interpretar el espacio, la Historia y el patrimonio cultural y natural del lugar en el que vivimos y hacen pensar al alumnado sobre la complejidad de las dinámicas pasadas que han dado lugar a esa realidad, así como sobre la obligación de ser partícipe en los mismos de forma responsable, cívica y creativa (López, Mora, Arrebola, \& Medina, 2017).

\section{METODOLOGÍA}

En consonancia con los aspectos que desarrollaremos en las siguientes líneas, el presente artículo expone una investigación con metodología mixta (con preguntas abiertas y cerradas) sobre la percepción que tiene el alumnado de la enseñanza de la didáctica de la historia local de la ciudad de Córdoba a través de la visita al museo arqueológico realizada por los dos grupos de tercer curso del Grado de Educación Primaria de la asignatura de Didáctica de las Ciencias Sociales, compuesto por unos 112 alumnos, de la Facultad de Ciencias de la Educación de la Universidad de Córdoba durante los cursos académicos 2019/2020.

El proceso metodológico que hemos creído más conveniente para desarrollar esta actividad fue participativo, flexible, partiendo de la práctica del aula para establecer una serie de etapas, las cuales tuvieron delimitadas sus funciones correspondientes.

En una primera fase, se comenzó trabajando en el diseño de las actividades con el objetivo de aplicar una metodología orientada a la mejora de ejercicio docente y del propio profesorado y alumnado, con una visión dinámica de la actividad. Se procedía a la búsqueda bibliográfica, restringir el objeto de enseñanza-aprendizaje y la selección de una muestra significativa. El objetivo se fundamentó en el conocimiento de las distintas dimensiones históricas-temporales, a través de una visita didáctica al Museo Arqueológico de Córdoba.

Seguidamente, se revisó en clase el Decreto 97/2015, de 3 de marzo, por el que se establece la ordenación y las enseñanzas correspondientes a la Educación Primaria en Andalucía. Comprendía casi en exclusiva los contenidos del área de Ciencias Sociales - 
Bloque 4: Las huellas del tiempo y Bloque 3: El mundo en el que vivimos- y, por supuesto, en los objetivos referidos al conocimiento de la historia de Andalucía y España a lo largo de los diferentes periodos históricos.

Se dispuso que el alumnado, en grupos compuestos por entre 4-6 integrantes, examinara la información del currículum y seleccionara aquella que se ajustará más a la temática del ejercicio propuesto, tratando de justificar una fundamentación teórica para la realización de la visita museística. Un aspecto que, sin lugar a dudas, fomentó el enriquecimiento de la actividad educativa fue la preparación y planificación previa de los itinerarios. Igualmente se propuso analizar una selección de artículos, webs... que complementara la información y el conocimiento ofrecido.

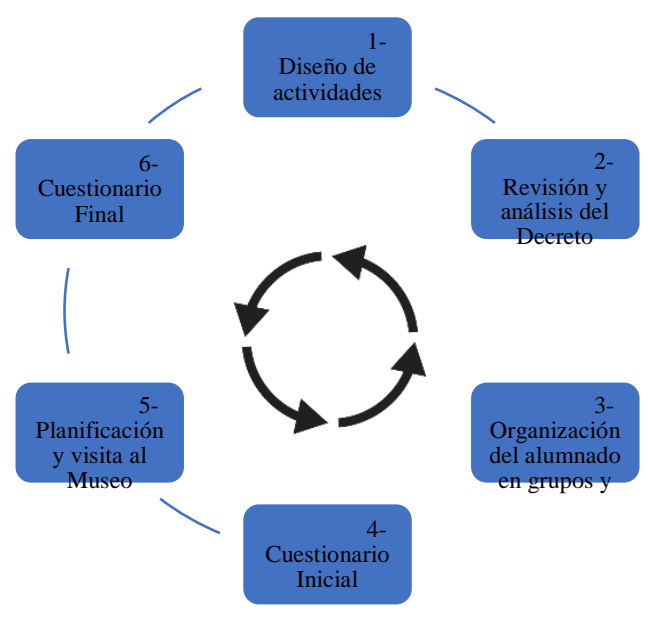

Figura 1. Secuenciación de actividades (Elaboración propia).

Junto a estos aspectos, se acompañó una tarea de investigación previa y una puesta en común en el aula como primer paso para conocer las realidades patrimoniales de nuestro entorno más cercano; igualmente nos ayudamos de una serie de herramientas informáticas, cuestionarios realizados con la plataforma Google Forms, para evaluar los posibles errores y mejoras de cara a una futura optimización académica del recurso. Creemos también que ha sido un extra de motivación para nuestro alumnado el poder desarrollar la actividad en el lugar pudiendo ver, tocar (en algunos casos) y experimentar unas sensaciones que en el aula no se pueden reproducir.

Una vez que el alumnado estuvo familiarizado con los contenidos del área, se procedió a la elaboración y aplicación de un cuestionario inicial cuya finalidad comprendía una primera evaluación diagnóstica que nos serviría de pauta para comprobar el nivel de conocimientos, opiniones y preferencias que los y las estudiantes poseían de cuestiones relativas al patrimonio histórico, museos y formación didáctica patrimonial, entre otras.

La siguiente etapa, consistió en planificar una visita en grupos pequeños disponiendo libertad total para su configuración, es por ello, que seleccionaron sin atender a ninguna motivación particular, salvo su afinidad personal o grado de amistad. Se 
proyectaron en el aula imágenes del privilegiado enclave en el que está ubicado el museo y se analizaron los tres bloques temáticos en los que está organizado: 1- Córdoba y su territorio 2- Córdoba, poder y centralidad 3- Vivir en Córdoba.

Realizada la actividad, se procedió a la recogida y análisis de la información a través de un cuestionario de evaluación final compuesto de un total de 30 preguntas. Incluye los 112 cuestionarios recibidos; la codificación de los datos y en las preguntas de contenido abierto a su respectivo análisis. Estaba conformado por cuatro bloques temáticos (I. Formación en patrimonio. II. Itinerario didáctico. III. Desarrollo competencial. IV. Museo arqueológico y la didáctica.) con un total de treinta preguntas.

A través de estas herramientas se midió el grado de adquisición de conocimientos antes de la visita, tras la realización de la misma, así como la asistencia y participación en actividades relacionadas con el patrimonio histórico local más allá del ámbito universitario. El cuestionario inicial estaba compuesto por diez preguntas de respuestas cortas y cuatro en las que se debía reflejar el grado de relevancia, teniendo en cuenta que un valor de 1 significaba poco relevante y un valor de 5, muy relevante. La encuesta final se realizó una vez completada la salida al Museo Arqueológico y, como anteriormente hemos comentado, está compuesto de treinta preguntas de respuestas cortas y largas intercaladas con cuestiones de tipo valorativo, asignando al valor 1 un significado de muy en desacuerdo y al valor 5 muy de acuerdo.

\subsection{Objetivos}

Una vez que estuvieron bien delimitadas y estructuradas las fases de la actividad, se formularon una serie de objetivos generales y específicos:

\section{Objetivos Generales:}

- Plantear estrategias metodológicas que pongan en valor la didáctica del patrimonio para la enseñanza de la historia local en el aula de Educación primaria, a través de las visitas o itinerarios al museo arqueológico de Córdoba

\section{Objetivos específicos:}

- Evaluar el comportamiento de los alumnos y alumnas utilizando un recurso como las visitas museísticas como elemento transmisor de contenidos.

- Confirmar si la metodología pedagógica aplicada ha sido eficaz en el aula para captar la atención del alumnado a la hora del aprendizaje de contenidos de naturaleza más teórica como pueden ser los relacionados con el currículum y la historia local.

- Analizar los niveles de motivación e interés que provocan los itinerarios patrimoniales en el alumnado de Educación Primaria.

El examen y análisis de la información se llevó a cabo a través de técnicas que permitieron los análisis descriptivos, estadísticas y gráficos. Para ello, se utilizó el 
paquete estadístico SPSS, versión 25.0 para Windows que facilitó la obtención de los siguientes resultados.

\section{RESULTADOS}

Los itinerarios didácticos son una herramienta pedagógica que mejoran el proceso de enseñanza-aprendizaje de las Ciencias Sociales, a la vez, que estimulan positivamente al alumnado a la hora de enfrentarse con contenidos de naturaleza teórica, como son los conceptos históricos o patrimoniales, por medio de una alta motivación y una activa participación que propicia que se fomente la creatividad y el pensamiento crítico y social.

Las variables de identificación nos indican que predomina el género femenino, con 73 mujeres $(65,2 \%)$, frente al masculino, con 39 varones, lo que supone un $(34,8 \%)$ del total de los y las participantes.

Con respecto a la edad, comprobamos en las respuestas que la mayoría se encuentran entre los 20 y 23 años (81,3\%); siendo minoritaria la representación de estudiantes mayores de este intervalo de edad.

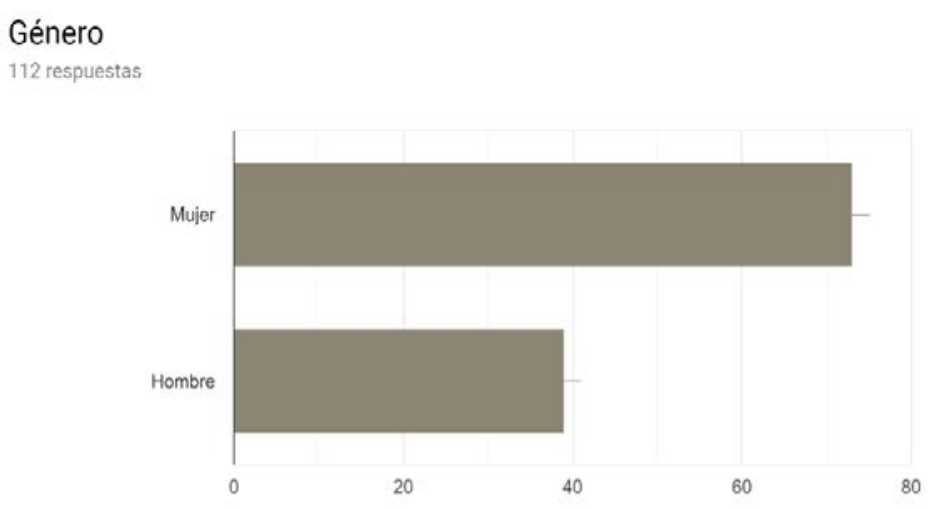

Figura 2. Resultados de la variable de identificación.

Sobre los datos correspondientes al contexto, de los 112 alumnos y alumnas que componen dicha muestra, el 70.6\% tienen su lugar de residencia en Córdoba capital, por lo que deducimos que dichos estudiantes deben de estar familiarizados con el patrimonio local de la ciudad. El resto porcentual se divide entre poblaciones de la provincia: Bujalance, Montoro, Fuente Palmera, Priego de Córdoba, Villanueva de Córdoba, Montilla, Villa del Río... y una cuota casi residual de personas (5.4\%) que tiene su residencia fuera de la provincia: Albacete, Sevilla y Cádiz, entre otras localidades.

Una de las preguntas formuladas en el cuestionario iniciales la concerniente a la idea que tiene nuestro alumnado sobre el concepto de patrimonio; se les preguntó que escribieran cuatro palabras que tuvieran relación con él. Se trataba de indagar el nivel de conocimientos previos de los estudiantes implicados en esta actividad. 
De las 112 respuestas, destaca sobremanera, la palabra historia aparece casi por unanimidad, seguida de otras como: monumentos, museos, conocimiento, antigüedad y cultura. Es reseñable que un número escaso de alumnos y alumnas no identificaran el término patrimonio con identidad, tradición, ideología, costumbres, orgullo, herencia. Con los resultados obtenidos, observamos cómo existe un elevado número de estudiantes que no sienten la historia local como parte de su identidad personal, es por ello que asimilan su aprendizaje en una práctica docente memorística como forma de estudio de la historia.

Esta imagen de metodología tradicional provoca un rechazo en el alumnado hacia estas disciplinas al considerarlas aburridas, carentes de motivación y de nula utilidad práctica en su cotidianidad académica, laboral y personal.

Al hilo de la cuestión anterior, planteamos una de naturaleza similar para incidir en el grado de conocimientos previos sobre las materias abordadas, en este caso hacíamos referencia a la utilidad de los museos. Algunas respuestas significativas fueron las siguientes:

- Para conservar restos y obras de arte que muestran cómo era la vida y el arte en el pasado, para que observemos las diferencias y evoluciones que se han producido.

- Para poder presenciar momentos históricos pasados que no pudimos presenciar en su día y, además, aprender de otra forma que no sea siempre dentro de un aula.

- Para mostrar a toda la sociedad, cualquiera puede visitarlos, distintas formas de arte actual, antigüedades que no tienen por qué ser expresiones artísticas, sino objetos cotidianos que se conservan, en un museo también se observa la evolución que ha habido a través de las épocas... si todo este patrimonio se encuentra en museos está protegido y bien conservado, independientemente de la índole del museo.

- Para mostrarnos cómo era el pasado.

Respecto a las cuestiones formuladas, una de las que creemos más importantes es la relativa a la formación recibida sobre patrimonio a lo largo del grado de Educación Primaria. Atendiendo a los resultados obtenidos, podemos comprobar que existe un elevado porcentaje de estudiantes, 67,9 (76) que afirman haber recibido formación sobre patrimonio e historia local, mientras que el resto de los encuestados responden todo lo contrario $32,1 \%$ (36). 
112 respuestas

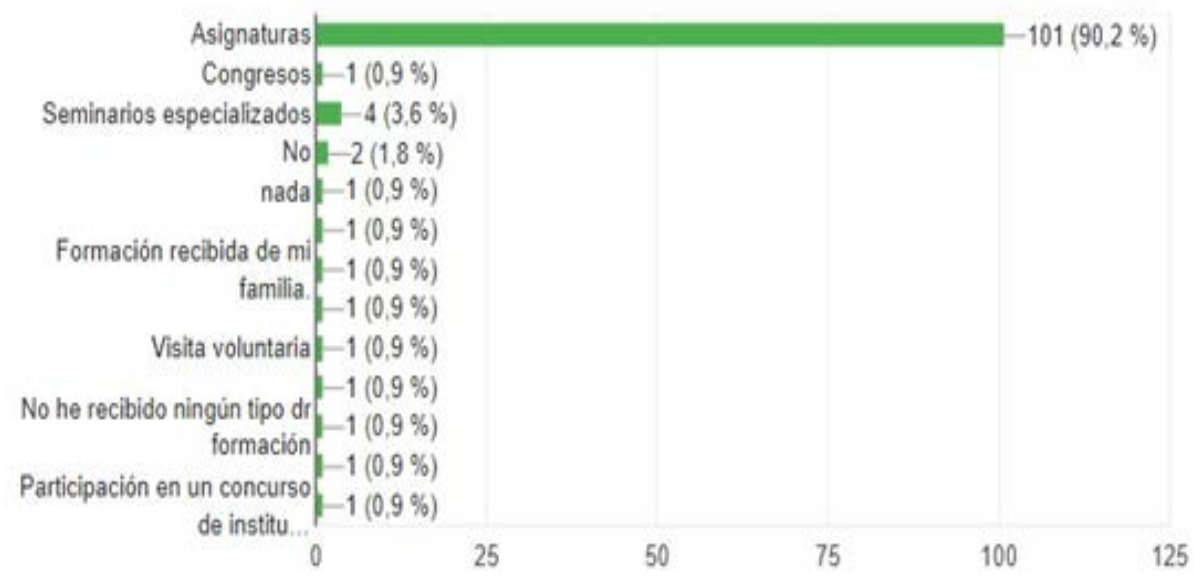

Figura 3. Tipo de formación recibida en patrimonio. (Elaboración propia)

Destacamos que, a lo largo de los cuatro años del grado de Educación Primaria de la Universidad de Córdoba, no existe una asignatura específica que verse sobre el patrimonio histórico, por lo que, consideramos que las respuestas se fundamentan en base a las asignaturas optativas cursadas por el alumnado. Sus respuestas reflejan que la formación no es específica y depende de los contenidos que cada docente incluya en asignaturas que puedan ser afines. Es por ello extraño, que más del $67 \%$ de los participantes hayan afirmado haber recibido una buena formación en patrimonio histórico a lo largo del grado de Educación Primaria, mientras que el 32,1\% contestaron lo contrario.

Creemos que la respuesta anterior viene influenciada por la formación que ha recibido nuestro alumnado en etapas anteriores como Secundaria o incluso Primaria. Se le interpelaba sobre este hecho, pero centrado las tareas de aprendizaje en el aula de Ciencias Sociales. A la vista de los resultados, el alumnado defiende que la enseñanza del patrimonio debe estar presente en todos los niveles del sistema educativo español.

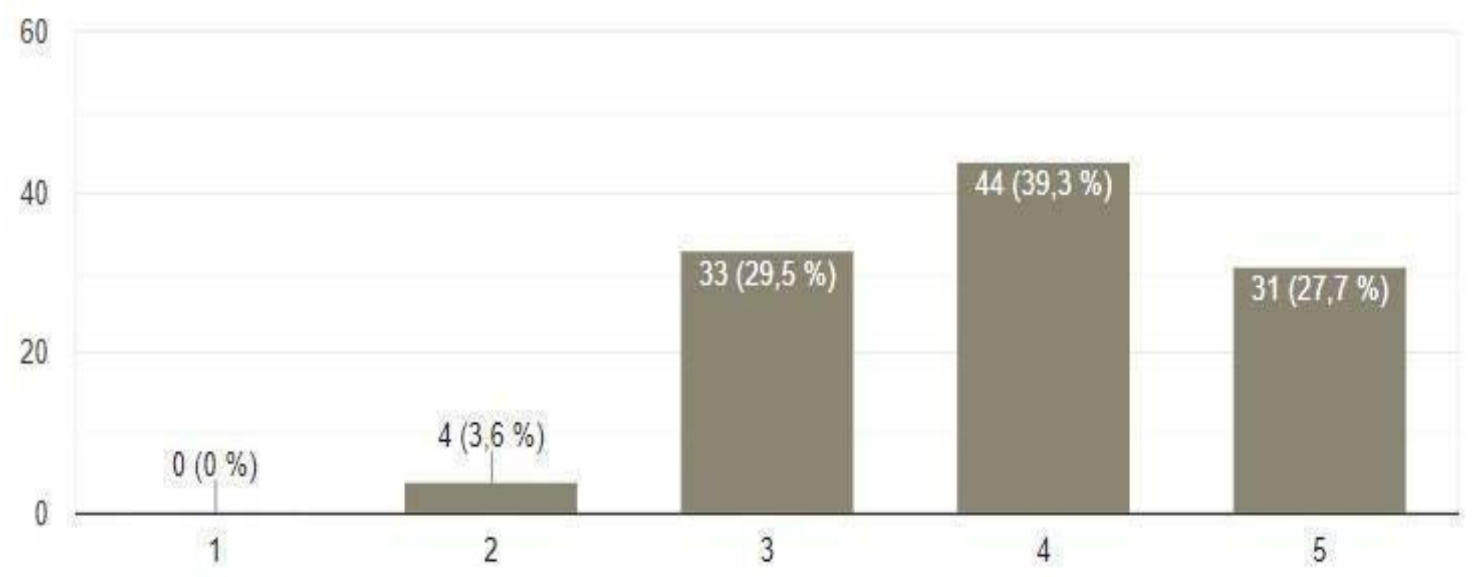


Figura 4. La visita al museo arqueológico puede contribuir a fomentar la identidad individual. (Elaboración propia).

Además, son conscientes de la necesidad que tienen ellos mismo de comprender qué significa el patrimonio histórico para su formación personal y del acercamiento de los niñas y niñas en la etapa de Educación Primaria. Por lo tanto, en palabras de Fontal, Ibáñez-Etxeberria y Martínez (2017), muchos estudiantes conocerán los bienes culturales y sus características, pero no lograrán que ese bien cultural se convierta en su propio patrimonio, ni mucho menos lograrán identificarse con él.

Con la intención de incidir más en el tratamiento didáctico y en el modo en el que los planes de estudios abordan el patrimonio, realizamos sendas preguntas para calibrar la percepción que sobre la cuestión tienen los futuros docentes. El gráfico que recoge la información derivada del análisis (Fig. 5) muestra como el 48.2\% (54 estudiantes) están de acuerdo en que las visitas o los itinerarios didácticos permiten trabajar contenidos no explícitos en el currículum de Primaria, el 37, 5\% se mostraban aún más favorables. Solo el $1.8 \%$ de los encuestados declararon no estar de acuerdo con la cuestión, mientras que el $12.5 \%$ se posicionaron de manera neutral.

Así mismo, las puntuaciones referidas a las dos cuestiones que se les planteaban en relación a si los itinerarios son recursos pedagógicos válidos para trabajar la transversalidad y la historia desde una perspectiva de género, arrojaron claros porcentajes favorables. En el caso de la primera, un 95.5\% de los estudiantes afirmaron estar de acuerdo en que la metodología empleada en la actividad fomenta el trabajar contenidos de forma transversal. Por la tanto deducimos que, salvando grandes cuestiones, el alumnado detecta un cierto interés general hacia la historia local y el patrimonio histórico como elemento capaz de vertebrar la comprensión de las identidades, el conocimiento de técnicas y recursos, y como fomentador de actitudes, dentro de la transversalidad.

Igualmente, en respuesta a la cuestión sobre género, 93 estudiantes (83.1\%) se mostraron a favor de poder utilizar las visitas al museo arqueológico para explicar la historia o el patrimonio con perspectiva de género. Hoy día se hace del todo innegable la importancia que tiene para una transformación social real el hecho de incluir la perspectiva de género en la formación del profesorado, para que en el futuro ejerza su función docente teniendo en cuenta dicha perspectiva. De manera residual, 3 estudiantes (2.7\%) mostraron su disconformidad con la cuestión. El 14.3\% se manifestaron neutrales sin saber si con este tipo de recursos didácticos podrían ser de utilidad para fomentar la perspectiva de género.

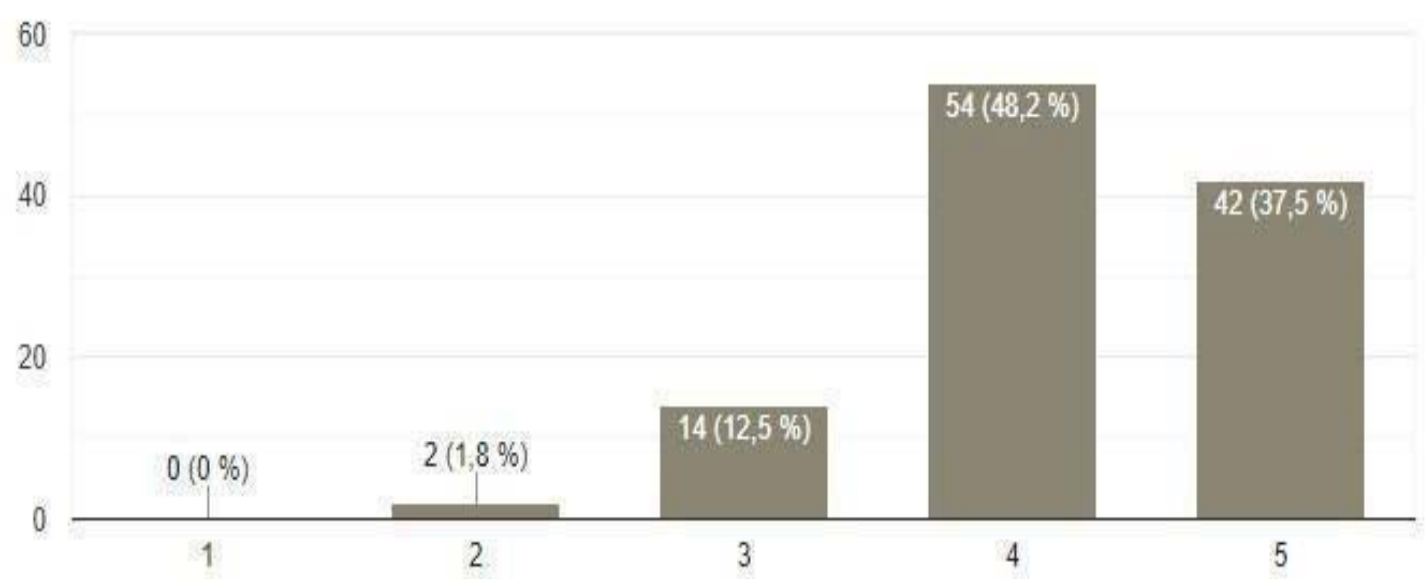


Figura 5. El itinerario en el museo arqueológico permite trabajar contenidos no explícitos del currículum. (Elaboración propia).

En lo concerniente a la preparación previa de la visita al museo arqueológico se presentaron a los y las estudiantes diferentes opciones para comprobar si, efectivamente, se habían involucrado en su propio proceso de aprendizaje mediante la búsqueda de información en la web del museo u otros recursos digitales, en monografías, artículos, etc... Los resultados muestran diferencias significativas debido al mayor atractivo que tiene entre el alumnado los contenidos de carácter práctico, frente a las actividades más teóricas que con mayor frecuencia se realizan en el aula.
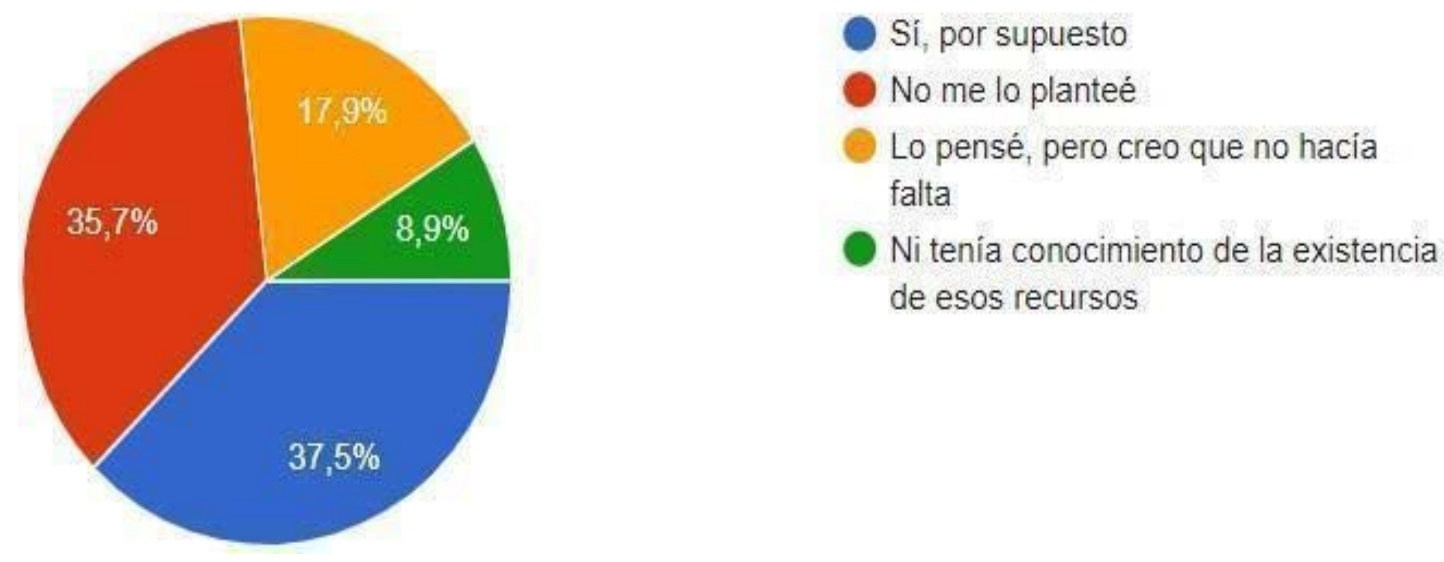

Figura 6. Resultados sobre la preparación previa al itinerario didáctico (Elaboración propia).

Con respecto a la variable temática sobre la formación, intentamos resolver la pregunta: ¿entiendes la historia de la ciudad de Córdoba de otra manera? una vez realizada la visita propuesta en el ejercicio. Su respuesta la encontramos muy fragmentada (Fig. 6): casi un $40 \%$ de nuestro alumnado se inclina positivamente ante la utilidad de la visita didáctica, ya que considera que entiende mejor la historia de su ciudad, para un 33\% la actividad le ha servido como motivación para complementar los conocimientos que atesoraba e incentivar la búsqueda de información sobre el patrimonio local; el 27, 7\% hacen hincapié en que tras la realización de la práctica prestaran más relevancia a cuestiones vinculadas con nuestro legado histórico.

Solo el 3,6\% tiene una percepción negativa de la actividad o puntualiza que la formación recibida en el itinerario por el museo no le ha sido provechosa para entender de manera diferente la historia de Córdoba.

\section{LIMITACIONES DEL ESTUDIO}

A pesar de los resultados positivos recabados con la investigación dedicada a la historia local y el museo arqueológico de Córdoba, surgen diferentes problemáticas que 
nos hacen reflexionar sobre qué caminos transitar para el desempeño de una mejor enseñanza de las Ciencias Sociales con estas herramientas.

Por una parte, y tal como afirma Joaquim Prats, la incorporación de la historia local al aula es mucho más complicada de lo que puede parecer. En muchas ocasiones, hay quien entiende que su enseñanza está relacionada con un concepto simplista de qué ocurrió en mi pueblo o quién o para qué se construyó determinado edificio o monumento. En definitiva, una acumulación de datos y fechas que no aportan nada relevante a la enseñanza de la Historia. Por este motivo, puede ser interesante que el docente tenga una cierta experiencia investigadora en el pasado, o que tenga un cierto interés en el lado científico de esta disciplina, que ayude a un mejor desarrollo del proceso de enseñanzaaprendizaje. Por otro lado, esta experiencia serviría para una mejor inmersión por parte del alumnado en el método de investigación, punto principal para fomentar la pedagogía del descubrimiento (Prats, 1996).

Por otro lado, es necesario que existan unos buenos recursos que ayuden a desarrollar la enseñanza de la historia local que nos lleve al conocimiento de una historia total y nos aleje de una comprensión simplona del conocimiento histórico. En el caso que estamos mostrando en estas páginas, la utilización del museo arqueológico con fines pedagógicos puede resultar un recurso del todo satisfactorio, tanto por su estrecha vinculación con la educación del patrimonio como por su labor por conservar y difundir los vestigios del pasado de la localidad. Sin olvidarnos tampoco del interés mostrado por los museos por expandir su dimensión educativa.

Sin embargo, es necesario acondicionar adecuadamente los contenidos para que sean accesibles y comprensibles al alumnado. Por una parte, a pesar de los esfuerzos en este campo, muchos de los museos no están del todo acondicionados para desarrollar una visión didáctica de su contenido. Esto puede resultar un problema que solamente se puede resolver con una previa y concienzuda preparación del itinerario didáctico y de este modo evitar que resulte una actividad aburrida o una mera visita turística sin trasfondo educativo a nuestro alumnado.

$\mathrm{Y}$ es que, en general, los museos son espacios complejos que hacen difícil su entendimiento. En su seno se custodian piezas de distintas etapas pretéritas, que en la mayoría de las ocasiones el espectador, de primeras, no logra entender en toda su dimensión. Por este motivo, es inevitable unas nociones previas, conocimientos que ayudarán a los discentes a interpretarlos y darles su sentido, tanto en su contexto histórico como en su dimensión en la sociedad actualidad. Sólo de este modo seremos capaces de explotar todo su potencial didáctico, en especial en todo lo que tiene que ver con el patrimonio (Ávila y Duarte, 2014).

Respecto a la educación patrimonial, existe un recurrente problema relacionado con la formación del profesorado en este aspecto. Y es que, en relación con la educación superior, un reciente estudio muestra la insuficiente presencia del patrimonio en la formación de los futuros maestros y maestras de Primaria (Fontal, Ibañez, Martínez y Rivero, 2017: 79-95). En general, como no se han programado asignaturas que aborden la enseñanza del patrimonio específicamente, su docencia solamente está reunida en materias optativas, como ocurre en las universidades públicas, con lo que el alumnado que no decida matricularse en ellas siempre presentará dificultades para alcanzar amplias 
competencias en contenidos patrimoniales y a los relacionados con su didáctica. En este sentido, tampoco se familiarizará con los elementos del patrimonio de su ciudad o comunidad, dejando de lado estos recursos didácticos, tan importantes para enseñar el proceso histórico más cercano, el del medio donde desarrollan sus vidas como ciudadanos.

\section{CONCLUSIONES Y DISCUSIÓN DE RESULTADOS}

Por los resultados recogidos podemos afirmar que el alumnado de la asignatura de Didáctica de las Ciencias Sociales del Grado de Primaria de la Universidad de Córdoba ha aceptado positivamente la práctica docente relacionada con el museo arqueológico de su ciudad. A través de la mismas, se han convencido de que los museos no son sólo espacios de conservación y exposición del patrimonio histórico, bien relacionados el turismo, sino que también son un valioso recurso didáctico que pueden utilizar en el futuro, cuando se desempeñen como profesores y profesoras (Bonilla Martos, 2017). Incluso algunos alumnos y alumnas habían visitado en alguna ocasión dicho museo y no habían llegado a estas conclusiones.

En este sentido, también comprenden, como bien lo expresan en el cuestionario, que para realizar cualquier actividad en el museo es necesario que el docente elabore a priori, junto con el alumnado, un trabajo de preparación, motivación y búsqueda de información, para que se facilite la explotación del máximo provecho de la experiencia didáctica.

Más allá del estudio y enseñanza del patrimonio, de gran valor para la Didáctica de las Ciencias Sociales, el alumnado ha aprendido sobre la historia de la ciudad de Córdoba a través de la actividad que se les ha planteado, y especialmente durante la realización del itinerario desarrollado por el museo. Según sus respuestas, lo han conseguido de forma amena, entretenida y diferente y eso ha sido posible gracias a las características temáticas de la exposición del museo arqueológico, fundamentadas cronológica y temáticamente.

Este artículo muestra que la experiencia pedagógica llevada a cabo por los estudiantes de tercero de la asignatura de Didáctica de las Ciencias Sociales, aún debe de profundizar en muchos aspectos para que podamos hablar de que los itinerarios son, a día de hoy una realidad educativa en la mayoría de los centros escolares de la ciudad de Córdoba. Comprobándose que los principales argumentos esgrimidos en estas líneas hayan sido: la falta de una formación específica en patrimonio a lo largo del grado, una baja motivación a la hora de acometer el estudio de disciplinas eminentemente teóricas como la historia y el desconocimiento del patrimonio más cercano, así como de los museos. No obstante, es un elemento ilusionante el que los futuros profesores encaren con motivación las metodologías y objetivos presentados de la historia local y el patrimonio más cercano.

Es del todo necesario intervenir para llevar a efecto el cambio de esta situación, ya que son muchas y variadas opciones pedagógicas para que cualquier centro educativo normalice las visitas o itinerarios didácticos a los museos de la ciudad, como una opción factible para la enseñanza-aprendizaje de las Ciencias Sociales. Este cambio puede 
facultarse desde la universidad, en los grados de Educación, tal y como se muestra en este trabajo, fomentando el conocimiento de las metodologías necesarias para organizar el itinerario escolar, incitando la motivación del alumnado universitario de los grados de educación a participar en la metodología y la planificación de este recurso. Así, creemos que nuestro alumnado se familiarice, y pueda poner en práctica en un futuro cuando ejerzan profesionalmente en sus centros educativos el itinerario o las visitas como herramienta para el conocimiento de nuestra historia local y patrimonio para que pase a ser una realidad educativa en las escuelas.

Así pues, con los resultados obtenidos de esta práctica fuera del aula, creemos que los estudiantes han modificado su percepción algo negativa sobre el estudio de las Ciencias Sociales, valorando positivamente que hay diversas prácticas docentes atractivas para el estudio de disciplinas eminentemente teóricas como la historia, geografía o el patrimonio.

Por último, indicar que creíamos un objetivo fundamental a la hora de plantear un ejercicio de estas características, el inculcar a los docentes de nuestro futuro más cercano, que en última instancia deben de entender que las visitas a los museos son parte fundamental tanto para la consecución de un aprendizaje significativo como para su formación personal y didáctica.

\section{REFERENCIAS BIBLIOGRÁFICAS}

Aguado Molina, M. (2018). Aportaciones de la museología al aprendizaje de los futuros maestros de E. Infantil sobre Didáctica específica de las Ciencias Sociales. En Buscando formas de enseñar: investigar para innovar en la didáctica de las Ciencias Sociales, López Torres, E. et al. (eds.), 233-242. Valladolid: Universidad de Valladolid.

Aranda, A.; Del Pino, M. J.; Montés, F. (2010). Los aspectos patrimoniales en la educación primaria en la nueva reforma educativa de Andalucía (España). Revista Iberoamericana de Educación, 52 (1).

Ávila, R. M. y Duarte, O. (2014). “Mi patrimonio, mi museo”. Una experiencia didáctica en la formación inicial del profesorado de Educación Infantil. En Una mirada al pasado y un proyecto de futuro: investigación e innovación en didáctica de las ciencias sociales, Pagès, J. y Santisteban, A. (coords.). Barcelona: AUPDCS.

Ballesteros, E., et al. (coords.) (2003). Patrimonio y la didáctica de las Ciencias Sociales. Cuenca: AUPDCS.

Belarte Franco, M.C. (2004), Historia, museografía y didáctica: Conversaciones con Joan Santacana, ÍBER: Didáctica de las ciencias sociales, geografía e historia, 39, 7-16.

Blanco Lozano, P., Ortega Gutiérrez, D., Santamaría, J. (2003). El Patrimonio y la Didáctica de las Ciencias Sociales. En El Patrimonio y la Didáctica de las Ciencias Sociales, Ballesteros, E. et al. (coords.), 71-80. Ediciones de la Universidad de Castilla-La Mancha Asociación Universitaria de Profesores de Didáctica de las Ciencias Sociales.

Bonilla Martos, A. L. (2017). Los museos como recurso didáctico: El ejemplo de los museos arqueológicos. Antropología Experimental, 17, Texto 21, 297-303. 
Clio '92 (1995). Tesisulladidatticadellastoria, Per la conoscenzadellestorielocalinellascuola, Tesisulcurricolidellascuola elementare, Quaderno 1, aprile 2000. www.storiairreer.it.

Cuenca, J. M. (2002). El patrimonio en la didáctica de las ciencias sociales: análisis de concepciones, dificultades y obstáculos para su integración en la enseñanza obligatoria. (Tesis Doctoral). Huelva: Universidad de Huelva.

Estepa Giménez, J., Jiménez Pérez, R., Martín Cáceres, M.J., Wamba Aguado, A.M., Cuenca López, J.M. (2012), La educación patrimonial: ¿qué y cómo se enseña en el museo y en la escuela? En El patrimonio cultural y natural como motor de desarrollo: investigación e innovación, Peinado Herreros, A. (coord.), 1274-1286. Jaén: Universidad Internacional de Andalucía.

Fernández, M. (2003), Los museos: espacios de cultura, espacios de aprendizaje, ÍBER: Didáctica de las ciencias sociales, geografía e historia, 36, 55-61.

Fontal Merillas, O., Ibáñez-Etxeberria, A., Martínez Rodríguez, M. \& Rivero Gracia, P. (2017). El patrimonio como contenido en la etapa de Primaria: del curriculum a la formación de maestros. Revista Electrónica Interuniversitaria de Formación del Profesorado, 20 (2), 79-95.

Fontal, O. (2013). El patrimonio en la escuela. Más allá del patrimonio como contenido curricular. En La educación patrimonial. Del patrimonio a las personas, O. Fontal (coord.), 23-44. Gijón: Trea.

(2016). El patrimonio a través de la educación artística en la etapa de primaria. Arte, Individuo y Sociedad, 28(1), 105-120.

Friera Suárez, F. (2003). Itinerarios didácticos: teoría y experiencias en defensa del Patrimonio. En El patrimonio y la Didáctica de las Ciencias Sociales, C. Fernández Fernández, J. A. Molina Ruiz, P. Moreno Benito (coords.), 339-346. Universidad de Castilla-La Mancha.

García de Miguel, J.M. (2012), El Patrimonio Histórico como memoria colectiva. En El patrimonio cultural y natural como motor de desarrollo: investigación e innovación, Peinado Herreros, A. (coord.), 56-74. Jaén: Universidad Internacional de Andalucía.

García Díaz, P. \& Martín Marcos, P. (2009), Museos arqueológicos de Gijón: el patrimonio arqueológico como recurso didáctico, ÍBER: Didáctica de las ciencias sociales, geografía e historia, 59, 53-63.

Garcia Ruiz, A. (1993) Los trabajos de campo: Visitas de Estudios Itinerarios Didácticos. Didáctica de las Ciencias Sociales en la Educación Primaria. Sevilla: Algaida.

Gesché-Koning, N. (1998), Museos y enseñanza de las ciencias sociales: Perspectivas de futuro, ÍBER: Didáctica de las ciencias sociales, geografía e historia, 15, 7-14.

Gil Carmona, F. (2018). Museos y formación del pensamiento social en educación primaria una propuesta de intervención didáctica. (Tesis doctoral). Barcelona: Universitat Autónoma. https://www.tdx.cat/handle/10803/650279.

González, N.; Pagès, J. (2003). El Patrimonio y la Didáctica de las Ciencias Sociales. En El Patrimonio y la Didáctica de las Ciencias Sociales, Ballesteros, E. et al. (coords.), 123-134. Ediciones de la Universidad de Castilla-La Mancha Asociación Universitaria de Profesores de Didáctica de las Ciencias Sociales. 
Hernández Cardona, F.X. (2005). Museografía didáctica. En Museografía didáctica, Serrat Antolí, N., Santacana Mestre J. (coords.), 23-61. Madrid: Ariel.

López Arroyo, C.T. (2014). La enseñanza del patrimonio a partir del entorno próximo a los centros educativos. ÍBER: Didáctica de las ciencias sociales, geografía e historia, 78, 61-71.

López Serrano, M.J. \& Guerrero Elecalde, R. (2019). La didáctica de la historia local de Córdoba en educación primaria a través del museo arqueológico. En Investigación, Innovación docente y TIC. Nuevos horizontes educativos, S. Alonso et al. (eds). 2077-2089. Madrid: Dykinson, S.L.

Lucas Palacios, L. \& de Alba Fernández, N. (2017), Educación patrimonial para formar ciudadanos críticos. ÍBER: Didáctica de las ciencias sociales, geografía e historia, 89, 26-31.

Marín López, R. (2003). Notas sobre el concepto de Historia e Historia Local. II Congreso Historia Local de Metodología de la Investigación Histórica. La Orotava. Gobierno de Canarias.

Martínez, M. (2016). Análisis comparativo de la imagen del patrimonio en la legislación nacional y autonómica, dentro de la educación obligatoria. En Patrimonio, identidad y ciudadanía en la enseñanza de las ciencias sociales, S. Molina, A. Escribano y J. Díaz (eds.), 186-199. Murcia: Universidad de Murcia.

Olave Farías, D. (2005). El itinerario didáctico: una propuesta metodológica para el análisis geo-histórico local. Geo-enseñanza, $10,2$. http://www.redalyc.org/pdf/360/36010206.pdf.

Pagès, J. (2000). La formación inicial del profesorado para la enseñanza del patrimonio histórico y de la historia. Treballs d' Arqueologia, 205-217.

Pedreño Ros, D. (2015). Aportaciones Didácticas de la Historia Local y Familiar al Proceso de Enseñanza-Aprendizaje de las Ciencias Sociales. Experiencias en Tres Centros Educativos de la Región de Murcia de Educación Primaria, Secundaria y PCPI. (Tesis doctoral). Murcia: Universidad de Murcia. http://hdl.handle.net/10201/46981

Pineda-Alfonso, J.A. (2018), Ciudadanía y trabajo con escalas. ÍBER: Didáctica de las ciencias sociales, geografía e historia, 93, 29-34.

Poblet Romeu, M.J. (2004). El patrimonio histórico, una herramienta para la enseñanza de la historia. IBER: Didáctica de las ciencias sociales, geografía e historia, 40, 116-122

Pol Méndez, E. (2001), Aprendiendo a percibir la ciudad: Metodología y estrategias didácticas, ÍBER: Didáctica de las ciencias sociales, geografía e historia, 27, 4148.

Prats, J. (1996). El estudio de la Historia local como opcióndidáctica. ¿destruir o explicar la historia. IBER Didáctica de las ciencias sociales, geografía. e historia, 8.

Rico Cano, L.; Ávila Ruiz, R.M. (2003). Difusión del Patrimonio y educación. El papel de los materiales curriculares. Un análisis crítico. En El Patrimonio y la Didáctica de las Ciencias Sociales. Universidad de Castilla-La Mancha, Ballesteros, E. et al. (coords.), 31-40. Ediciones de la Universidad de Castilla-La Mancha Asociación Universitaria de Profesores de Didáctica de las Ciencias Sociales. 
Rivero, P. (2008). Propuesta para una integración del patrimonio arqueológico en el Nuevo currículo de Educación primaria. En Didáctica de las Ciencias Sociales, currículo escolar y formación del profesorado. La didáctica de las Ciencias Sociales en los nuevos planes de estudio, R.M. Ávila, A. Cruz y M.C. Díez (eds.), 391-400. Jaén: Universidad de Jaén / Asociación universitaria de profesorado de Didáctica de las Ciencias Sociales.

Serrano Moral, C. (2018), Qué ocurrió cuando visitamos el museo con las manos fuera de los bolsillos... En Buscando formas de enseñar: investigar para innovar en la didáctica de las Ciencias Sociales, López Torres, E. et al. (eds.), 801-811. Valladolid: Universidad de Valladolid.

Valbuena Barrasa, M. (1994), El patrimonio cultural, testigo de una época. ÍBER: Didáctica de las ciencias sociales, geografía e historia, 2, 61-78. 